\title{
Effect of the cooling sensation induced by olfactory stimulation by L-menthol on dyspnoea: a pilot study
}

\author{
To the Editor:
}

It is common for us to encounter patients suffering from dyspnoea who can occasionally obtain subjective relief when they sit near an open window or in front of a fan. In fact, a recent randomised controlled study showed the effectiveness of a handheld fan blowing air across the nose and mouth in reducing the sensation of breathlessness in patients with advanced disease [1]. It has also been reported that, in patients with chronic obstructive pulmonary disease, breathing cold air reduces the sensation of dyspnoea and improves exercise performance [2]. However, the mechanism behind this effect remains unclear.

Transient receptor potential melastatin 8 (TRPM8) channels are gated by cold temperature [3]. Expression of the TRPM8 gene has been observed in trigeminal and vagal afferent neurons [4]. Recent studies and reviews have provided a solid basis showing that L-menthol also acts on the TRPM8 channels in peripheral sensory neurons [5-7]. L-menthol-evoked activation of TRPM8 channels induces a cooling sensation, and respiratory peripheral afferent input from TRPM8 stimulation is thought to play a key role in monitoring flow in the airway [8]. However, although facial or nasal airflow seems to have an important role in reducing dyspnoea, the effect of the L-menthol-evoked cooling sensation on exertional dyspnoea has not yet been fully elucidated. As it is recognised that the perception of dyspnoea results partly from neuroventilatory uncoupling [9], which is a mismatch of the command to breathe and the mechanical response, alterations to the sensory afferents via the activation of TRPM8 may represent a novel therapeutic opportunity for the treatment of dyspnoea induced by exercise.

Exercise-induced dyspnoea often occurs during various activities of daily living in diverse respiratory diseases [10], and it is known to be modulated by peripheral sensory afferents in skeletal muscle [11, 12]. Like any other sensation, the intensity of dyspnoea during exercise is determined by a combination of peripheral afferent inputs and central gain amplification [13]. However, it has not been clarified whether olfactory stimulation of TRPM8 alters exercise-induced dyspnoea. In the present study, we analysed the effect of olfactory stimulation by L-menthol (OSM) on dyspnoea induced by constant-load exercise. As there is evidence that the activation of peripheral afferents caused by locomotor muscle contraction can augment the perception of dyspnoea [14], the state of the peripheral sensory input that is involved in dyspnoea during inspiratory resistive load breathing may be different from that in exertional dyspnoea. In addition, sustained resistive load breathing is often used for inspiratory muscle training, which would evoke dyspnoea. We therefore simultaneously investigated the perception of dyspnoea during external inspiratory resistive loads.

25 healthy subjects (mean \pm SD $21.6 \pm 2.1$ years old; 11 female; body mass index: $21.7 \pm 2.6 \mathrm{~kg} \cdot \mathrm{m}^{-2}$ ) were enrolled in our randomised, single-blind, placebo-controlled, crossover study. The subjects came to our laboratory for 5 days within a 2 -week period, and each experimental session was separated by a 24-h interval. During the first visit, the subjects were given a short training period to accustom them to the apparatus and the use of the modified Borg scale. Then, the subjects performed a symptom-limited incremental cycle exercise test to determine the exercise intensity corresponding to an oxygen consumption $\left(V^{\prime} \mathrm{O}_{2}\right)$ of $80 \%$ of the anaerobic threshold ( $80 \%$ AT-watts). During the exercise, $V^{\prime} \mathrm{O}_{2}$, carbon dioxide production, minute ventilation, breathing frequency and tidal volume were measured by using a facemask connected to a metabolic analyser. OSM was administered by using an L-menthol-scented patch. The placebo patch had a strawberry odour. These patches, which contained monocomponent L-menthol or strawberry scent (Harou-herb, Toko Industry, Ebetsu, Hokkaido, Japan), were attached to the inside of 
a facemask. During the second and third sessions, pulmonary function test measurements were taken before and after $10 \mathrm{~min}$ of olfactory stimulation by placebo (OSP) or OSM, which was assigned in a randomised manner, followed by $10 \mathrm{~min}$ of constant-load cycling with OSP or OSM at the exercise intensity corresponding to the $V^{\prime} \mathrm{O}_{2}$ at $80 \%$ AT-watts. During the fourth and fifth sessions, dyspnoea was induced by introducing an inspiratory resistive load to the external breathing circuit, and was assessed using the modified Borg scale under the OSP or OSM condition, which was assigned in a randomised manner $[15,16]$. In brief, the sensation of dyspnoea was measured while the subject breathed through a Hans-Rudolph valve with a linear inspiratory resistance $(\mathrm{R})$ of $0,5,15,25$ or $35 \mathrm{cmH} \mathrm{H}_{2} \mathrm{O} \cdot \mathrm{L}^{-1} \cdot \mathrm{s}^{-1}$. The loads were presented in the order of increasing magnitude. Neither ventilation nor breathing pattern was controlled during the test. After breathing for $1 \mathrm{~min}$ at each level of resistance, the subject rated the sensation of dyspnoea (discomfort when breathing); the subjects were instructed to avoid rating non-respiratory sensations, such as headache or irritation of the pharynx. Comparisons were performed using the modified Borg score at each load $\left(\mathrm{R}=0,5,15,25\right.$ or $\left.35 \mathrm{cmH}_{2} \mathrm{O} \cdot \mathrm{L}^{-1} \cdot \mathrm{s}^{-1}\right)$. For each subject, the estimated dyspnoea Borg scores were plotted against the corresponding amounts of the resistive loads using a $\log -\log$ transformation. Because it is known that a linear relationship exists between the amount of the load and the dyspnoea Borg score on a log-log scale [13], the slope and intersection were determined by linear regression analysis on a log-log scale. Then, the log dyspnoea thresholds were estimated as the point of intersection with the $\mathrm{x}$-axis, using the linear regression equation of the log-log relationship between the amount of the inspiratory resistive load and the modified Borg score. All subjects provided written informed consent for participation, and the study was approved by the institutional review board.

All subjects completed the experiments without any difficulty or side-effects. They all described a cooling sensation in the nose and/or airway during each session with OSM, but not with OSP. None of the subjects perceived any unpleasant smells during OSM or OSP. There were no significant changes in pulmonary function in response to the 10-min OSM (forced expiratory volume in $1 \mathrm{~s}\left(\mathrm{FEV}_{1}\right): 4.20 \pm 0.80 \mathrm{~L}$ versus $4.12 \pm 0.78 \mathrm{~L}$; forced vital capacity (FVC): $4.71 \pm 0.82 \mathrm{~L}$ versus $4.69 \pm 0.83 \mathrm{~L}$; $\mathrm{FEV}_{1} / \mathrm{FVC}$ : $88.56 \pm 5.78 \%$ versus $88.72 \pm 5.01 \%)$ or OSP $\left(\mathrm{FEV}_{1}: 4.14 \pm 0.71 \mathrm{~L}\right.$ versus $4.15 \pm 0.72 \mathrm{~L}$; FVC: $4.62 \pm 0.81 \mathrm{~L}$ versus $4.71 \pm 0.81 \mathrm{~L}$; $\mathrm{FEV}_{1} / \mathrm{FVC}$ : $86.73 \pm 5.45 \%$ versus $86.76 \pm 5.42 \%$ ). The perception of dyspnoea during constant-load exercise revealed a significant interaction between the olfactory conditions (OSP and OSM) and the duration of exercise. As shown in figure 1a, exertional dyspnoea under the OSM condition was significantly lower than that under the OSP condition from $3 \mathrm{~min}$ after the start of the exercise to $1 \mathrm{~min}$ after the end of the exercise. There was, however, no significant difference in tidal volume, respiratory rate, minute ventilation, heart rate or $V^{\prime} \mathrm{O}_{2}$ between the OSM and OSP conditions (data not shown).

The perception of dyspnoea during external inspiratory resistive loading revealed a significant interaction between the olfactory conditions (OSP and OSM) and the external inspiratory resistive load $(\mathrm{p}<0.05)$. As shown in figure $1 \mathrm{~b}$, the modified Borg score during inspiratory resistive load breathing under the OSM condition was significantly lower than that under the OSP condition from $\mathrm{R}=25$ to $\mathrm{R}=35 \mathrm{cmH} \mathrm{H}_{2} \mathrm{O} \cdot \mathrm{L}^{-1} \cdot \mathrm{s}^{-1}$. The log-log slope between the inspiratory resistive load and the dyspnoea Borg score was estimated for each subject. As shown in figure $1 \mathrm{c}$, there was a significant difference between the log-log slopes of the OSP and OSM conditions $(\mathrm{p}<0.05)$, suggesting that the central processing of respiratory-related signals in response to inspiratory resistive load breathing was downregulated by OSM. There was no significant
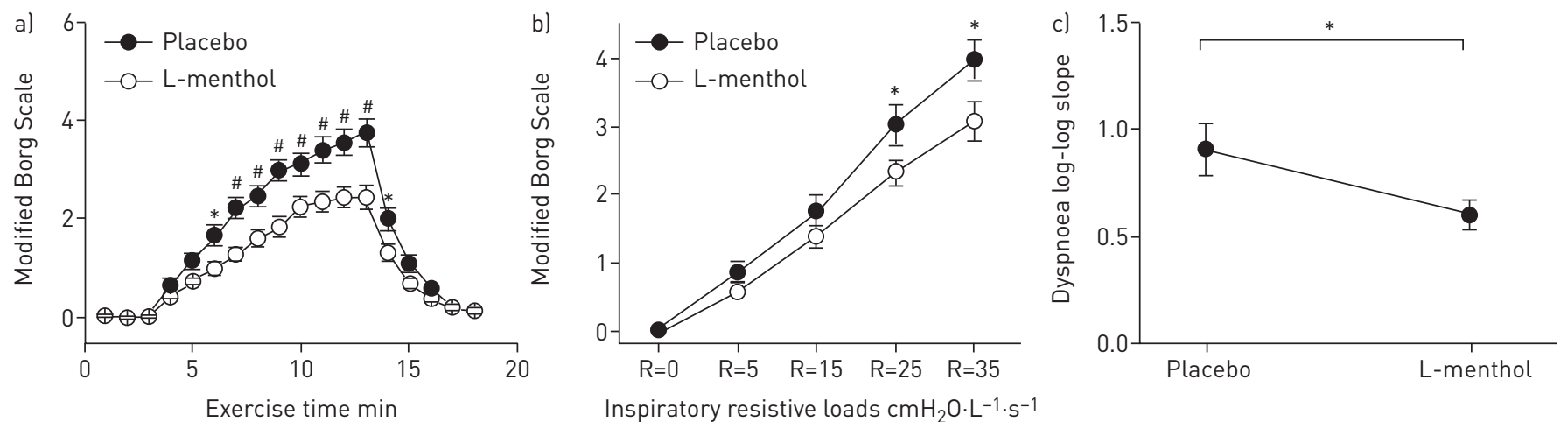

FIGURE 1 a) The comparisons in modified Borg scale during constant-load exercise between olfactory stimulation by placebo (OSP) and olfactory

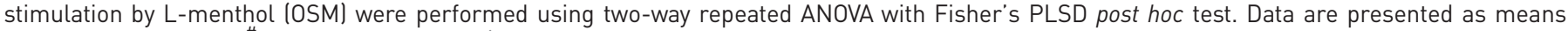

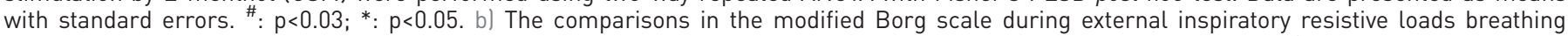
between OSP and OSM were performed using two-way repeated ANOVA with Fisher's PLSD post hoc test. *: p<0.05. c) Dyspnoea log-log slope calculated by the log-log linear regression when dyspnoea Borg scores were plotted as a function of resistive loads. Closed circles and error bars indicate the mean value and standard error. $p$-values were calculated by paired t-test. ${ }^{*}: p<0.05$. 
difference in the log dyspnoea threshold between the OSP and OSM conditions (data not shown); this indicated that L-menthol caused no significant changes in the peripheral sensors involved in unpleasant respiratory sensations induced by inspiratory resistive loads and that there is a larger contribution from central gain mechanisms than from peripheral ones.

The results of the present study suggest that OSM reduces the perception of dyspnoea induced by constant-load exercise and inspiratory resistive loads in normal volunteers. We also confirmed that OSM produced no side-effects.

Olfactory information ascends directly to the limbic system; therefore, odour induces immediate emotional changes and may also influence the respiratory rate and tidal volume. Several neuro-imaging studies found strong activation of the left amygdala and orbitofrontal cortices when subjects smelled highly aversive odours [17, 18]. In addition, ROYET et al. [19] revealed that unpleasant odours activated the piriformamygdala area and ventral insula more strongly than did pleasant odours. In the present study, no subject perceived the control or target olfactory stimulus as an unpleasant smell. In addition, no significant difference in respiratory rate or tidal volume during constant-load exercise was found between the OSP and OSM conditions, suggesting that the influence of OSM on respiratory changes may have been relatively modest in this study.

The underlying mechanism behind the inhibitory effects on dyspnoea of the cooling sensation from L-menthol may be mediated through a peripheral effect on TRPM8 and central neural mechanisms. The expression of TRPM8 has been observed in nasal trigeminal and vagal neurons [4]. In this study, L-menthol could be deposited onto the nasal and tracheal mucosa and lungs via odour. However, previous studies have shown minimal expression of TRPM8 on bronchopulmonary vagal afferents [3, 6, 8]. Moreover, Plevkova et al. [4] demonstrated that although L-menthol vapour markedly inhibited citric acid-evoked coughing, L-menthol had no effect on citric acid-evoked coughing when administered directly to the tracheal mucosa or when delivered as an aerosol to the intrapulmonary airways in anaesthetised guinea pigs. Thus, the respiratory reflex may be modulated by the activation of TRPM8 on nasal trigeminal neurons. As some pulmonary and airway sensory receptors and afferent pathways are common to both cough and dyspnoea [11], the activation of TRPM8 by L-menthol on nasal trigeminal endings may similarly play a key role in inhibiting dyspnoea.

It is possible that stimulation by L-menthol of TRPM8 on nasal trigeminal endings may also be the first step in eliciting a cognitive illusion of airway flow from the cooling sensation (i.e. cognition of an increased airway flow) that could improve the dissociation between the central ventilator drive and incoming afferent ventilator information from peripheral sensory nerves. On the other hand, it has been reported that menthol inhibits neurokinin A- and capsaicin-induced bronchoconstriction in vivo in guinea pigs [20]. However, in the present study, our observations were consistent with those of a past study revealing that the inhalation of menthol does not alter upper airway resistance in humans [21]. Therefore, it can be reasonably assumed that the cognitive illusion of airway flow from a cooling sensation can be associated with a reduction of dyspnoea from OSM during constant-load exercise and inspiratory resistive load breathing.

In conclusion, these results shed light on the possibility that the cooling sensation from OSM may provide an effective and safe pharmacological therapy that can be applied to relieve sensations of dyspnoea during both the static state and exertion.

Masashi Kanezaki ${ }^{1}$ and Satoru Ebihara ${ }^{2}$

${ }^{1}$ Dept of Physical Therapy, Faculty of Health Care Sciences, Himeji Dokkyo University, Himeji, Japan. ${ }^{2}$ Dept of Rehabilitation Medicine, Toho University Graduate School of Medicine, Tokyo, Japan.

Correspondence: Masashi Kanezaki, Dept of Physical Therapy, Faculty of Health Care Sciences, Himeji Dokkyo University, 7-2-1, Kamiohno, Himeji, Hyogo, Japan. E-mail: kmasashi@gm.himeji-du.ac.jp

Received: Sept 182016 | Accepted after revision: Dec 172016

Support statement: This study was supported by Grants-in-Aid for Scientific Research from the Ministry of Education, Culture, Sports, Science and Technology (26870760, 23659375, 24300187, 24659397 and 257166), Research Grant for Longevity Sciences from the Ministry of Health, Labor and Welfare (H22-Junkanki-shi-Ippan-001) and Research Funding for Longevity Sciences (22-2) from the National Center for Geriatrics and Gerontology (NCGG). Funding information for this article has been deposited with the Crossref Funder Registry.

Conflict of interest: None declared.

\section{References}

1 Galbraith S, Fagan P, Perkins P, et al. Does the use of a handheld fan improve chronic dyspnea? A randomized, controlled, crossover trial. J Pain Symptom Manage 2010; 39:831-838. 
2 Spence DP, Graham DR, Ahmed J, et al. Does cold air affect exercise capacity and dyspnea in stable chronic obstructive pulmonary disease? Chest 1993; 103: 693-696.

3 McCoy DD, Knowlton WM, McKemy DD. Scraping through the ice: uncovering the role of TRPM8 in cold transduction. Am J Physiol Regul Integr Comp Physiol 2011; 300: R1278-R1287.

4 Plevkova J, Kollarik M, Poliacek I, et al. The role of trigeminal nasal TRPM8-expressing afferent neurons in the antitussive effects of menthol. J Appl Physiol 2013; 115: 268-274.

5 McKemy DD, Neuhausser WM, Julius D. Identification of a cold receptor reveals a general role for TRP channels in thermosensation. Nature 2002; 416: 52-58.

6 Peier AM, Moqrich A, Hergarden AC, et al. A TRP channel that senses cold stimuli and menthol. Cell 2002; 108: 705-715.

7 Behrendt HJ, Germann T, Gillen C, et al. Characterization of the mouse cold-menthol receptor TRPM8 and vanilloid receptor type-1 VR1 using a fluorometric imaging plate reader (FLIPR) assay. Br J Pharmacol 2004; 141: 737-745.

8 Fisher JT. TRPM8 and dyspnea: from the frigid and fascinating past to the cool future? Curr Opin Pharmacol 2011; 11: 218-223

9 Jolley CJ, Luo YM, Steier J, et al. Neural respiratory drive and breathlessness in COPD. Eur Respir J 2015; 45: 355-364.

10 Castro AA, Porto EF, Iamonti VC, et al. Oxygen and ventilatory output during several activities of daily living performed by COPD patients stratified according to disease severity. PLoS One 2013; 8: e79727.

11 Davenport PW, Vovk A. Cortical and subcortical central neural pathways in respiratory sensations. Respir Physiol Neurobiol 2009; 167: 72-86.

12 Gagnon P, Bussieres JS, Ribeiro F, et al. Influences of spinal anesthesia on exercise tolerance in patients with chronic obstructive pulmonary disease. Am J Respir Crit Care Med 2012; 186: 606-615.

13 Gui P, Ebihara S, Kanezaki M, et al. Gender differences in perceptions of urge to cough and dyspnea induced by citric acid in healthy never smokers. Chest 2010; 138: 1166-1172.

14 Grippo A, Carrai R, Chiti L, et al. Effect of limb muscle fatigue on perception of respiratory effort in healthy subjects. J Appl Physiol 2010; 109: 367-376.

15 Kikuchi Y, Okabe S, Tamura G, et al. Chemosensitivity and perception of dyspnea in patients with a history of near-fatal asthma. N Engl J Med 1994; 330: 1329-1334.

16 Ebihara S, Ogawa H, Sasaki H, et al. Doxapram and perception of dyspnea. Chest 2002; 121: 1380-1381.

17 Zald DH, Pardo JV. Emotion, olfaction, and the human amygdala: amygdala activation during aversive olfactory stimulation. Proc Natl Acad Sci USA 1997; 94: 4119-4124.

18 Zald DH, Donndelinger MJ, Pardo JV. Elucidating dynamic brain interactions with across-subjects correlational analyses of positron emission tomographic data: the functional connectivity of the amygdala and orbitofrontal cortex during olfactory tasks. J Cereb Blood Flow Metab 1998; 18: 896-905.

19 Royet JP, Plailly J, Delon-Martin C, et al. fMRI of emotional responses to odors: influence of hedonic valence and judgment, handedness, and gender. Neuroimage 2003; 20: 713-728.

20 Wright CE, Laude EA, Grattan TJ, et al. Capsaicin and neurokinin A-induced bronchoconstriction in the anaesthetised guinea-pig: evidence for a direct action of menthol on isolated bronchial smooth muscle. $\mathrm{Br} J$ Pharmacol 1997; 121: 1645-1650.

21 Pereira EJ, Sim L, Driver HS, et al. The effect of inhaled menthol on upper airway resistance in humans: A randomized controlled crossover study. Can Respir J 2013; 20: e1-e4. 